\title{
Berberine ameliorates blockade of autophagic flux in the liver by regulating cholesterol metabolism and inhibiting COX2-prostaglandin synthesis
}

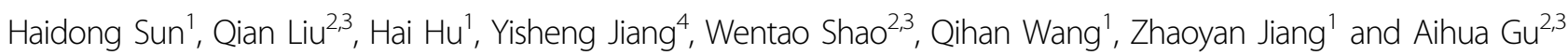

\begin{abstract}
Excessive cholesterol contributes to the development of cardiovascular diseases. Berberine (BBR) has been reported to regulate cholesterol homeostasis. Here, we found that BBR could ameliorate the hepatic autophagic flux blockade caused by cholesterol overloading. The underlying mechanism included lowering hepatic cholesterol level, modulating the cholesterol distribution targeting the plasma membrane by decreasing sterol carrier protein 2 expression and inhibiting cyclooxygenase 2-mediated production of prostaglandin metabolites, which decreased the phosphorylation of Akt/mTOR. Our study provides evidences that BBR could be a therapeutic agent for protecting liver under cholesterol overloading via the regulation of autophagic flux.
\end{abstract}

\section{Introduction}

Cholesterol overloading plays an important role in the development of metabolic disorders such as cardiovascular diseases ${ }^{1,2}$, which have been the leading cause of morbidity and mortality all over the world ${ }^{3,4}$. The liver is the key organ controlling cholesterol homeostasis in the body. Excessive cholesterol accumulation in hepatocytes may cause intensive lipotoxicity, such as endoplasmic reticulum (ER) stress ${ }^{5}$, lysosome impairment ${ }^{6}$, and mitochondrial function disruption ${ }^{7}$, and can further worsen metabolic disorders. Macroautophagy (referred to as autophagy) involves the formation of double-membrane autophagosomes that subsequently fuse with lysosomes for degradation ${ }^{8}$. Autophagy can participate in the

\footnotetext{
Correspondence: Aihua Gu (aihuagu@njmu.edu.cn) or Zhaoyan Jiang (zhaoyanjiang@gmail.com)

${ }^{1}$ Center of Gallbladder Disease, Shanghai East Hospital, Institute of Gallstone Disease, Tongji University School of Medicine, Shanghai, China

${ }^{2}$ State Key Laboratory of Reproductive Medicine, Institute of Toxicology, Nanjing Medical University, Nanjing, China

Full list of author information is available at the end of the article. These authors contributed equally: Haidong Sun, Qian Liu, Hai Hu Edited by G.M. Fimia
}

metabolism of lipids, glucose, and proteins ${ }^{9}$, providing energy under the conditions lacking sufficient nutrients, such as starving ${ }^{10}$. Emerging evidence has revealed that autophagy plays a crucial role in lipid balance ${ }^{11,12}$ and that impaired autophagic flux can cause excessive lipid accumulation, severe hepatic oxidative stress, and inflammation. Notably, excessive lipid accumulation may induce dysfunction of autophagy and the subsequent lysomal degradation.

Berberine (BBR), an isoquinoline alkaloid isolated from Berberis vulgaris L., is commonly used to treat diarrhea. In recent years, its roles in cholesterol level reduction ${ }^{13,14}$, anti-inflammation ${ }^{15}$, and anti-ER stress ${ }^{16}$ in hepatocytes have been reported. To date, the known mechanisms by which BBR lowers cholesterol include the upregulation of hepatic low-density lipoprotein receptor ${ }^{14}$ and the inhibition of intestinal cholesterol absorption ${ }^{17,18}$. BBR has also been reported to play a role in the regulation of autophagy in adipocytes ${ }^{19}$. Whether BBR has a similar effect in hepatocytes has not been clarified.

\section{(c) The Author(s) 2018}

(c) (i) Open Access This article is licensed under a Creative Commons Attribution 4.0 International License, which permits use, sharing, adaptation, distribution and reproduction cc) in any medium or format, as long as you give appropriate credit to the original author(s) and the source, provide a link to the Creative Commons license, and indicate if changes were made. The images or other third party material in this article are included in the article's Creative Commons license, unless indicated otherwise in a credit line to the material. If material is not included in the article's Creative Commons license and your intended use is not permitted by statutory regulation or exceeds the permitted use, you will need to obtain permission directly from the copyright holder. To view a copy of this license, visit http://creativecommons.org/licenses/by/4.0/. 
A
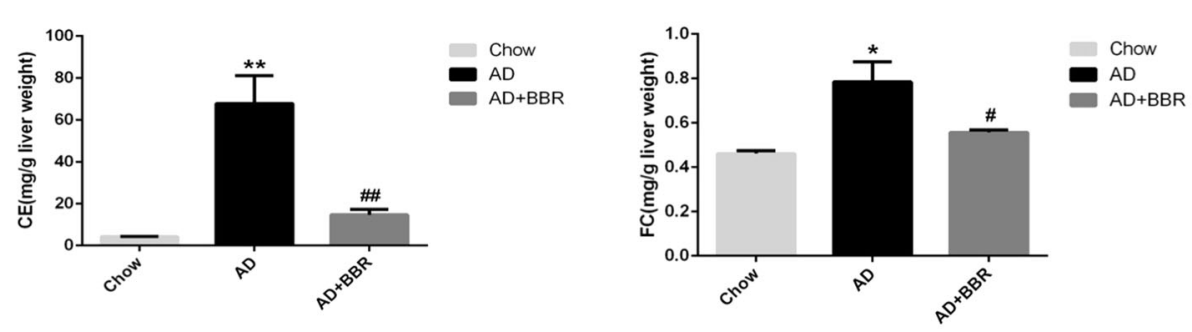

B
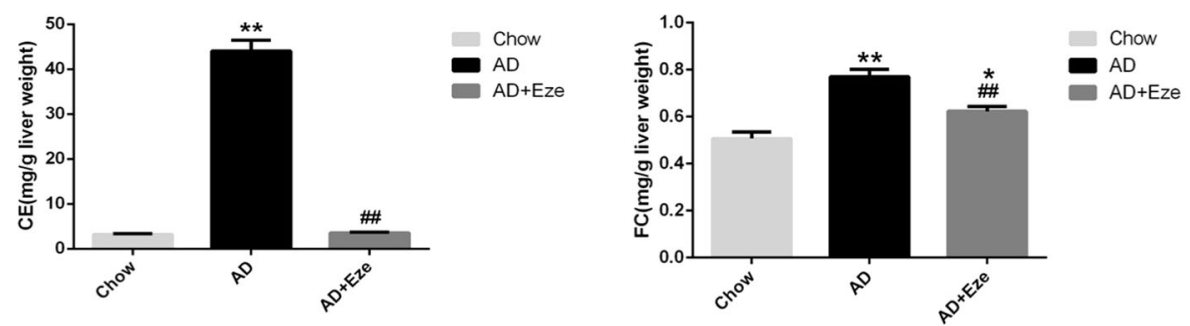

C

Chow

AD

$A D+B B R$

AD+Eze

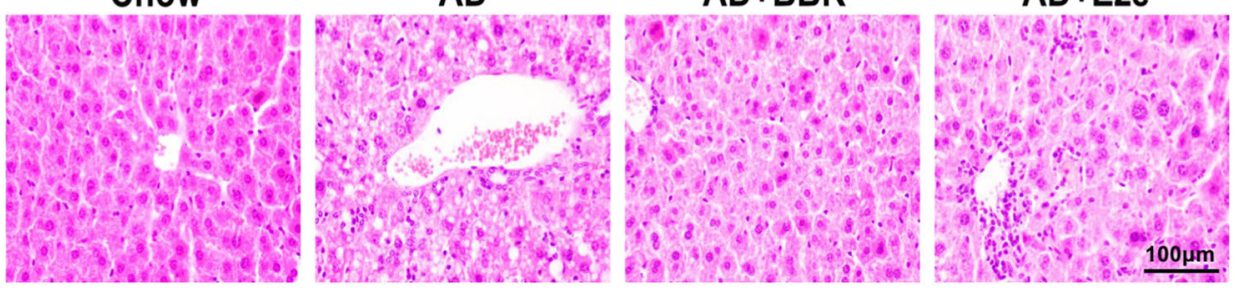

D

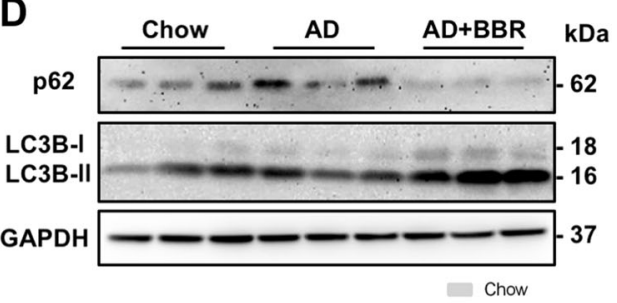

E
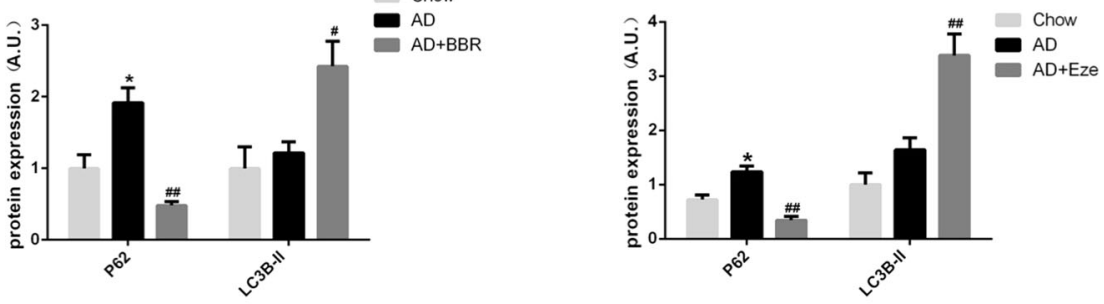

$\mathbf{F}$

LC

HC

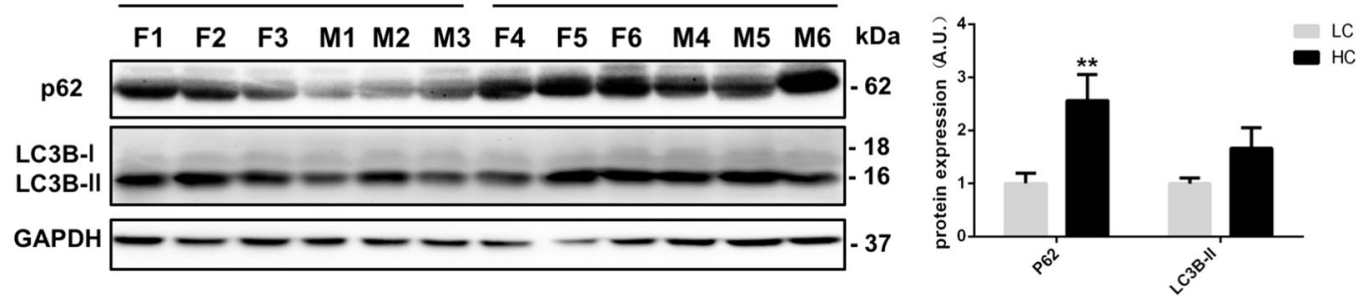

Fig. 1 (See legend on next page.) 
(see figure on previous page)

Fig. 1 Effect of BBR on hepatic cholesterol accumulation and blockade of autophagic flux. $\mathbf{a}, \mathbf{b}$ Cholesterol levels in the livers from mice fed with atherogenic diet (AD) or chow diet and treated with berberine (BBR) or ezetimibe (Eze). ${ }^{*} p<0.05$ and ${ }^{* *} p<0.01$ when compared with chow diet; $^{\#} p<0.05$ and ${ }^{\# \#} p<0.01$ when compared with AD. $\mathbf{c}$ H\&E staining of liver tissues from each group (magnification: $\times 40$ ). $\mathbf{d}, \mathbf{e} L \mathrm{LC} 3 \mathrm{~B}-\|$ and $p 62$ protein levels in livers from mice fed with chow diet, AD, or treated with BBR or Eze. ${ }^{*} p<0.05$ and ${ }^{* *} p<0.01$ when compared with chow diet; ${ }^{*} p<$ 0.05 and ${ }^{\# \#} p<0.01$ when compared with AD. f Hepatic LC3B-II and p62 protein levels between patients with high cholesterol level (HC) and those with low cholesterol level (LC) in their livers. ${ }^{* *} p<0.01$ for the HC group compared with the LC group

Free cholesterol (FC) accumulation in hepatocytes was shown to block autophagic flux ${ }^{20}$. In the present study, we found that BBR could reverse the blockade of autophagic flux caused by high cholesterol in hepatocytes. BBR reduced accumulation of hepatocellular cholesterol, inhibited the expression of sterol carrier protein 2 (SCP2), thus decreased cholesterol distribution toward plasma membrane (PM) and down-regulated cyclooxygenase 2 (COX2)-mediated prostaglandin (PG) metabolism. All these effects taken together, inhibited the phosphorylation of Akt/mammalian target of rapamycin (mTOR) signaling, which is a master regulatory pathway of autophagy process $^{21}$.

\section{Results}

BBR improved autophagic flux in the liver under cholesterol overloading

Atherogenic diet (AD; Fig. 1a) and high cholesterol diet (HCD; Figure S1A) led to the accumulation of cholesterol in mouse liver. BBR treatment dramatically lowered both FC and cholesteryl ester in mice fed with these diets (Fig. 1a and Figure S1A). A similar effect was produced following treatment with ezetimibe (Fig. 1b), a pharmaceutical compound that lowers the cholesterol level by effectively inhibiting intestinal Niemann Pick C1 like 1 (NPC1L1)-mediated cholesterol absorption ${ }^{22}$. Hematoxylin and eosin (H\&E) staining of mouse liver showed the appearance of hepatocyte ballooning due to the accumulation of lipid droplets that contained the bulk of $\mathrm{CE}$ and triglycerides (Fig. 1c). Both BBR and ezetimibe treatment improved the pathologic changes caused by AD.

We next examined the expression levels of two essential autophagy protein markers, microtubule-associated protein 1 light chain 3 (LC3) and SQSTM1 (p62). The accumulation of p62, indicating blockade of autophagic flux, was observed in the livers of mice fed with $\mathrm{AD}$ (Fig. 1d) as well as HCD (Figure S1B). Furthermore, in human liver biopsies (patient information listed in Supplementary Table 1), we also found blockade of autophagic flux in subjects who had high hepatic cholesterol content (Fig. 1f). In contrast, BBR treatment in mice significantly decreased the p62 protein level and increased the LC3B protein level (Fig. 1d). Similar findings were observed in mice treated with ezetimibe (Fig. 1e). These results suggested that BBR ameliorated the hepatic autophagic flux blockade related to cholesterol accumulation.

\section{BBR directly regulated autophagic flux in hepatic cells}

BBR treatment led to a significant increase in the appearance of green LC3B puncta in HepG2 cells (Figure S2A), suggesting the promotion of autophagosome/ autolysosome formation. With electron transmission microscopy, we also observed typical structures of doublemembrane formation, engulfment, and fusion with lysosomes and autolysosomes in the livers of mice treated with BBR (Figure S2B). The autophagic flux was further monitored in HepG2 cells expressing a tandem RFP-GFPLC3B fusion protein. The green signal of the GFP quenches under acidic conditions, which occur after fusion with lysosomes; the red signal of the RFP serves as a marker for autolysosomes; and puncta with merged signals, shown as yellow, represent autophagosomes ${ }^{23}$. Cholesterol treatment increased the number of yellow puncta in the merged images, which is a sign of impaired autophagosome fusion with lysosomes (Fig. 2a). A similar phenomenon was observed when these RFP-GFP-LC3B fusion protein-expressing HepG2 cells were incubated with chloroquine (CQ), a lysosomal inhibitor used to block autophagic flux. In contrast, when these cells were incubated with BBR, the RFP proportions of the merged images significantly increased, suggesting BBR could facilitate the fusion of autophagosomes with lysosomes.

Increases in the expression levels of both the LC3B and p62 proteins were observed in HepG2 cells incubated with cholesterol (Fig. 2b) or with CQ. BBR, in contrast, decrease the LC3B and p62 proteins in HepG2 cells incubated with cholesterol (Fig. 2c) or CQ (Fig. 2d).

The results of immunostaining further showed a significant accumulation of both the LC3B and p62 proteins when hepatocytes were loaded with cholesterol (Fig. 2e). The same results were found after CQ treatment. BBR significantly decreased the expression of LC3B and p62 in these cells (Fig. 2e).

\section{$B B R$ regulated the key proteins involved in cholesterol metabolism}

The main reservoir for cholesterol in cells is the PM, which may contain $35 \%$, and other intracellular organelles, such as the ER, may contain $5 \%$ of the total cholesterol $^{24}$. Filipin, which selectively binds to $\mathrm{FC}^{25}$, is 


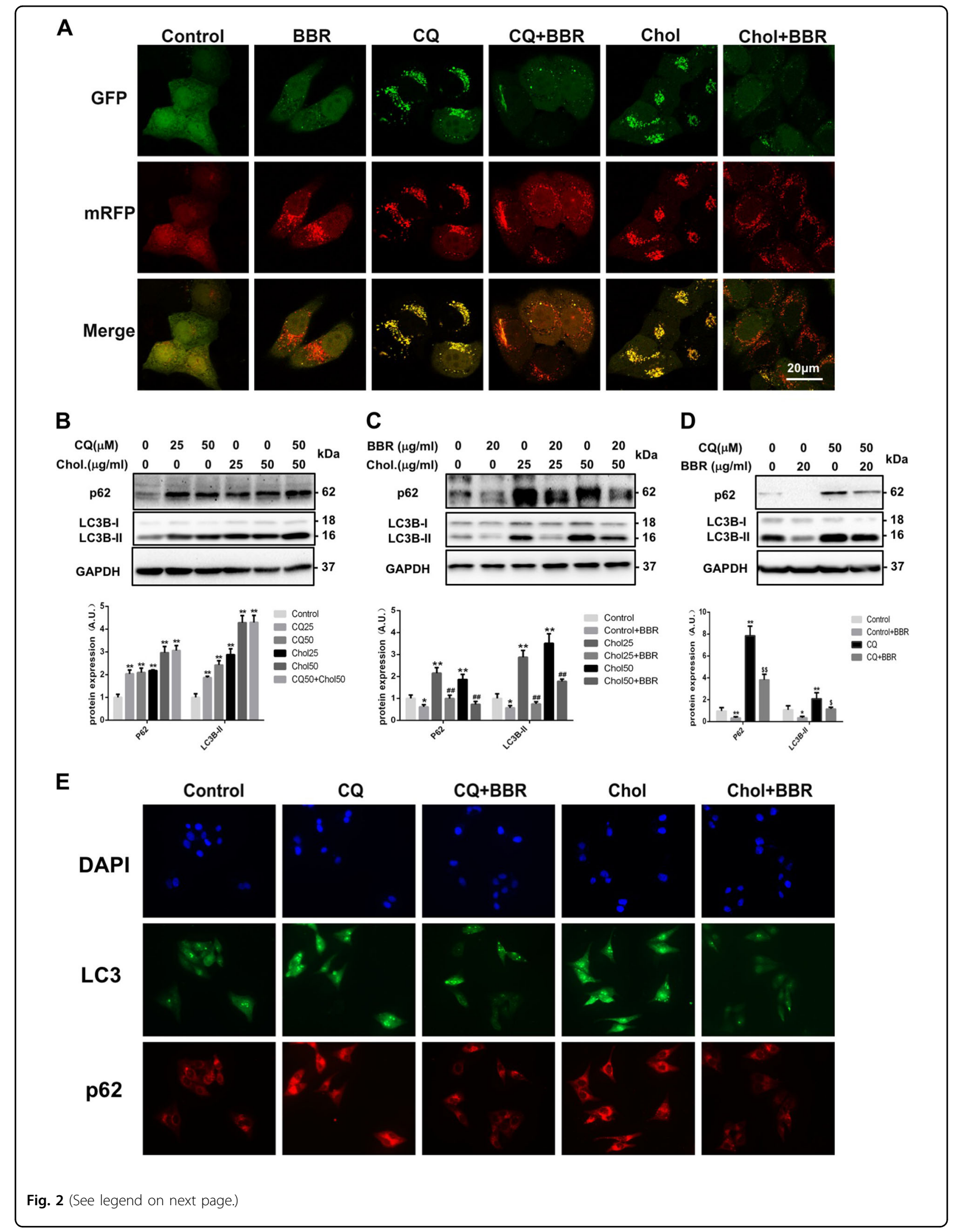


(see figure on previous page)

Fig. 2 Effect of BBR treatment on cholesterol-induced blockade of autophagic flux in HepG2 cells. a Confocal microscopy examination of HepG2 cells expressing a tandem GFP-RFP-LC3 fusion protein treated with cholesterol (Chol, $50 \mathrm{\mu g} / \mathrm{ml})$, chloroquine (CQ, 50 $\mu \mathrm{g} / \mathrm{ml})$, berberine (BBR, $20 \mathrm{\mu g} / \mathrm{ml}$ ), or their combinations for $24 \mathrm{~h}$. b LC3B-II and p62 protein levels in HepG2 cells treated with CQ, cholesterol (Chol), or a combination of CQ and cholesterol for $24 \mathrm{~h}$. c LC3B-II and p62 protein levels in HepG2 cells treated with cholesterol (Chol), berberine (BBR), or a combination of cholesterol and BBR for $24 \mathrm{~h}$. $\mathbf{d}$ LC3B-II and p62 protein levels in HepG2 cells treated with CQ, BBR, or their combination for $24 \mathrm{~h} .{ }^{*} p<0.05$; ${ }^{* *} p<0.01$ : compared with control. ${ }^{\# \#} p<0.01$ : compared with cholesterol at the same concentration. ${ }^{\$} p<0.05$ and ${ }^{\$ \$} p<0.01$ : compared with CQ. e Immunofluorescence staining of LC3B and p62 in HepG2 cells treated with cholesterol $(50 \mu \mathrm{g} / \mathrm{ml}), \mathrm{CQ}(50 \mu \mathrm{g} / \mathrm{ml}), \mathrm{BBR}(20 \mu \mathrm{g} / \mathrm{ml})$, or their combinations for $24 \mathrm{~h}$

applied to visualize the distribution of cholesterol in hepatocytes. Using fluorescence microscopy, we found filipin signals were prominent in the PM under basal conditions, but intracellularly, they were weak and diffuse (Fig. 3a). BBR treatment dramatically decreased the filipin signals in the PM and increased its co-localization with LAMP-1, a marker for late endosomes and lysosomes (Fig. 3a). These data suggested that BBR triggered the redistribution of intracellular FC.

Several transporter proteins are involved in intracellular cholesterol trafficking ${ }^{26}$. SCP2, the mature form of SCPX, is involved in the transport of cholesterol from the ER to the $\mathrm{PM}^{27}$. StAR-related lipid transfer domain containing 4 (STARD4) is involved in the efflux of cholesterol from late endosomes $^{28}$. Both proteins were down-regulated in HepG2 cells treated with BBR (Fig. 3b), also in cells treated with cholesterol (Fig. 3c). However, no difference in the expression of Niemann Pick C2 (NPC2), a protein involved in cholesterol trafficking between late endosomes and lysosomes ${ }^{29}$, was observed between cells treated with BBR and controls. The downregulation of hepatic SCP2 and STARD4 by BBR was further confirmed in mice fed with AD (Fig. 3d).

The conversion of cholesterol into bile acids is a crucial way to remove excessive cholesterol from the body. Cholesterol $7 \alpha$-hydroxylase (CYP7A1) is the rate-limiting enzyme for this process. Interestingly, we found that BBR dose-dependently upregulated the expression of CYP7A1 in HepG2 cells (Fig. 3b), in the presence or absence of cholesterol (Fig. 3c), and in mouse livers (Fig. 3d). Collectively, these data showed that BBR modulated cellular cholesterol by alleviating intracellular cholesterol trafficking targeting PM and by promoting the degradation of cholesterol into bile acids.

\section{BBR activated autophagy via inhibition of AKT/mTOR phosphorylation}

$\mathrm{AKT} / \mathrm{mTOR}$ is an important pathway regulating the autophagy process ${ }^{21,30}$. Our data showed that cholesterol overloading led to an activation of AKT and mTOR phosphorylation in HepG2 cells (Fig. 4a) as well as in human livers (Fig. 4d). Treatment with BBR inhibited the phosphorylation of both AKT and mTOR (Fig. 4b) and reversed the cholesterol-induced activation of AKT/
mTOR in both HepG2 cells (Fig. 4a) and mouse livers (Fig. 4c and Figure S1B). No alteration of ERK or AMPK phosphorylation by BBR was observed in the present study (data not shown). Our data indicated that BBR could induce autophagy through the inhibition of AKT/ mTOR phosphorylation.

AKT activation mainly takes place at the $\mathrm{PM}^{31}$, particularly at lipid raft sites where cholesterol is rich ${ }^{32}$. Using pervanadate, a potent AKT activator ${ }^{33}$, we observed localization and activation of AKT at the PM (Fig. 4e). Cholesterol loading also activated AKT phosphorylation at the PM, suggesting the recruitment of AKT to the PM. In contrast, $\mathrm{BBR}$ treatment attenuated the phosphorylation of AKT at the PM (Fig. 4e).

\section{BBR modulated autophagic flux through the inhibition of PGE2 synthesis}

A KEGG analysis of the RNA-seq data was performed to further unravel the potential mechanisms of BBR. Arachidonic acid (AA) metabolism was the pathway most regulated by $\mathrm{BBR}$ in mice fed with $\mathrm{AD}$ (Fig. 5a). The hepatic mRNA expressions of key genes involved in AA metabolism were increased by $\mathrm{AD}$ consumption and were down-regulated by BBR (Fig. 5b). Additionally, both the mRNA and protein levels of COX2 were increased by AD and lowered by BBR treatment (Fig. 5b, c). Target metabolomics analysis of the AA and PG metabolites revealed similar changes as $\mathrm{COX} 2$ expression in mice fed with $\mathrm{AD}$ and in those treated with BBR (Fig. 5d, e).

In cholesterol-overloaded HepG2 cells, the blockade of autophagic flux (Fig. 6a) was accompanied by an induction of COX2 expression (Fig. 6b). Treatment with celecoxib, a selective COX2 inhibitor, lowered the COX2 expression (Fig. 6c) and ameliorated the cholesterolinduced blockade of autophagic flux (Fig. 6a). Overexpression of COX2 in HepG2 cells led to significantly increased phosphorylation levels of AKT and mTOR (Fig. 6b), resulting in the blockade of autophagic flux (Fig. 6a). Treatment with BBR partly ameliorated this blockade (Fig. 6a) by inhibiting the expression of COX2 protein (Fig. 6b) and the phosphorylation of AKT/mTOR as well (Fig. 6d and Figure S3).

PGE2 comprises the majority of metabolites in PG metabolism and plays a vital role in hepatic inflammation 


\section{A}
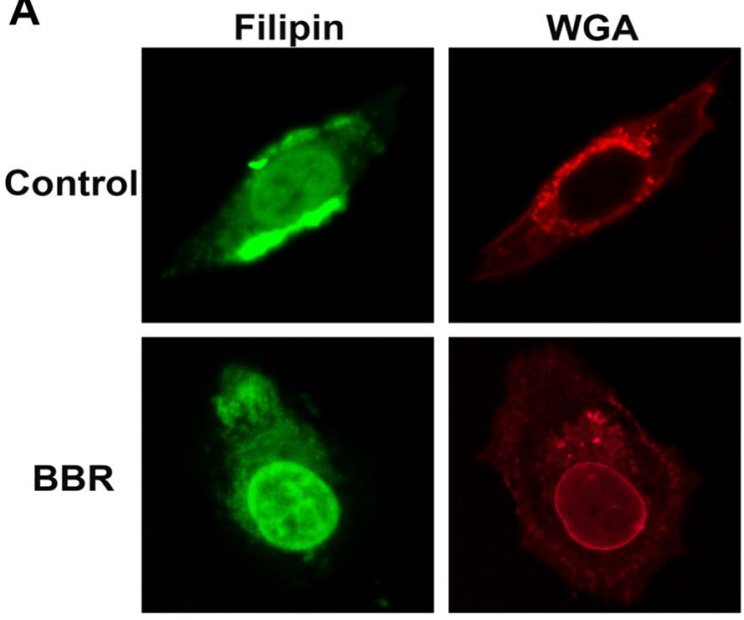

B

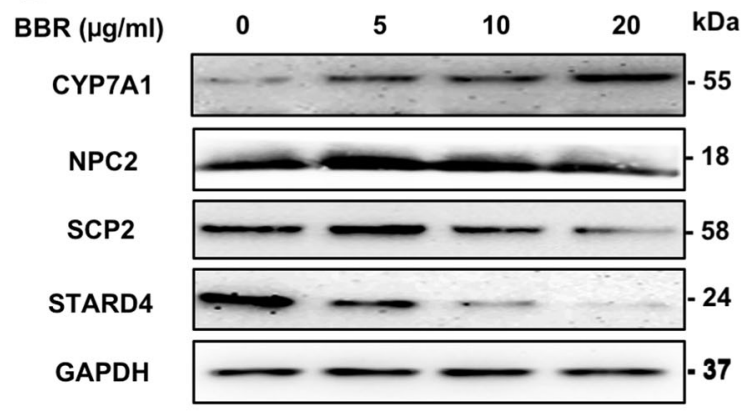

C

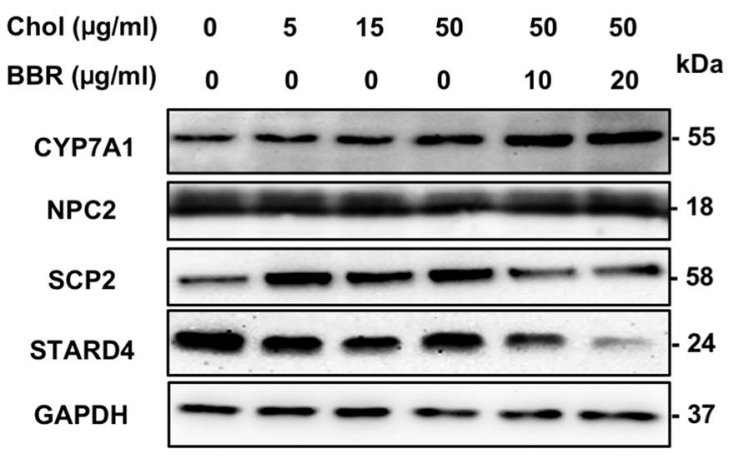

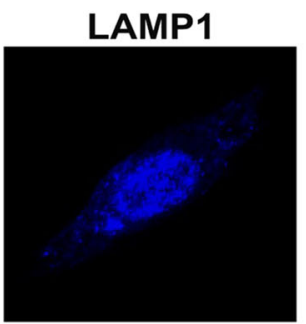
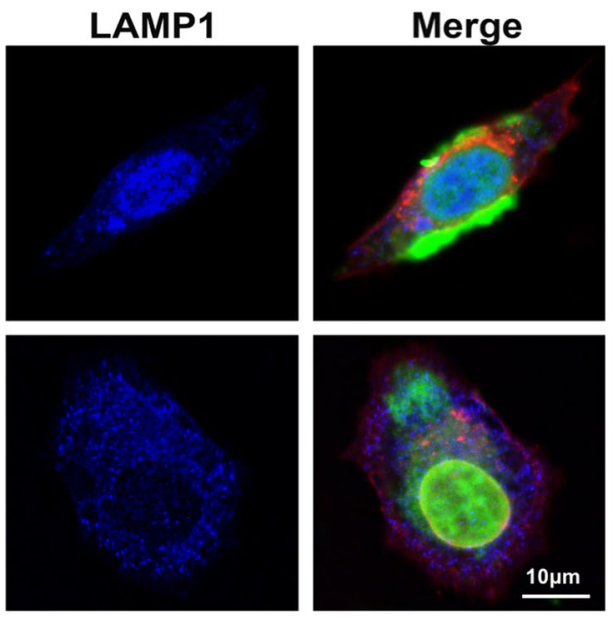

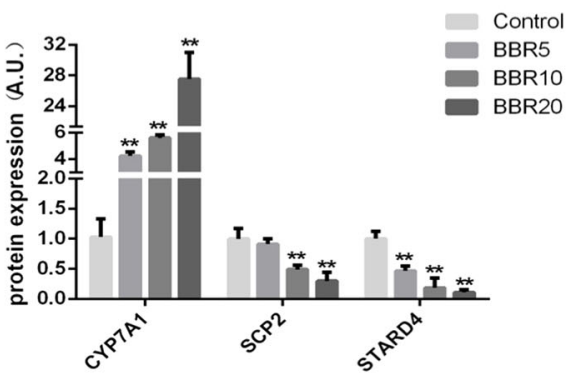

D
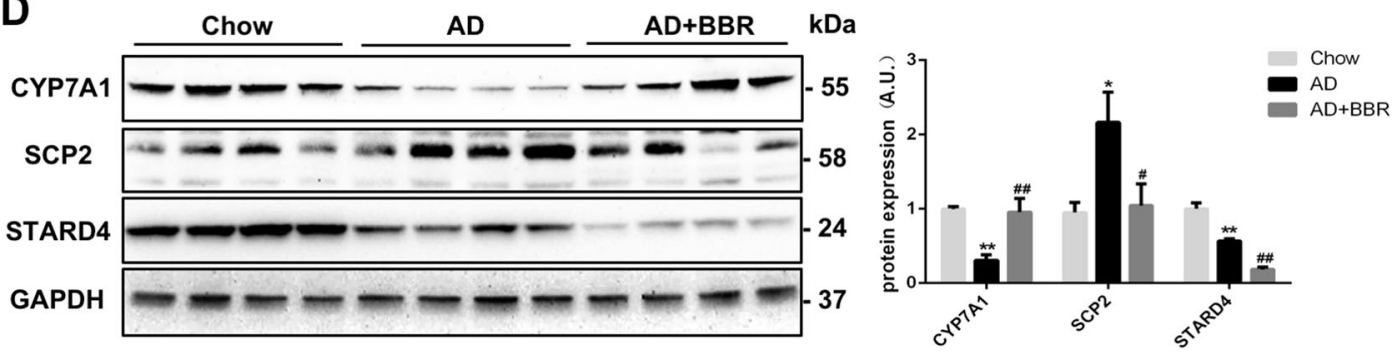

Fig. 3 (See legend on next page.) 
(see figure on previous page)

Fig. 3 Cholesterol distribution and transporter protein expression in BBR-treated hepatocytes. a Confocal microscopy staining of the cholesterol distribution in HepG2 cells treated with berberine (BBR, $20 \mu \mathrm{g} / \mathrm{ml}$ for $24 \mathrm{~h}$ ) as measured by filipin staining. Wheat germ agglutinin (WGA) and LAMP-1 were used for staining the membrane and lysosomes, respectively. $\mathbf{b}$ Expression of the CYP7A1, SCP2, STARD4, and NPC2 protein levels in HepG2 cells treated with different concentrations of berberine (BBR) for $24 \mathrm{~h}$. ${ }^{*} p<0.01$ compared with control. c Expression of the CYP7A1, SCP2, STARD4, and NPC2 protein levels in HepG2 cells treated with cholesterol alone (Chol) or cholesterol and BBR together for $24 \mathrm{~h} .{ }^{*} p<0.05$ and ${ }^{* *} p<$ 0.01 compared with control. ${ }^{\#} p<0.05$ and ${ }^{\# \#} p<0.01$ compared with cholesterol 50. d Expression of hepatic CYP7A1, SCP2, and STARD4 proteins in mice fed with different diets. ${ }^{*} p<0.05$ and ${ }^{* *} p<0.01$ compared with chow diet. ${ }^{*} p<0.05$ and ${ }^{\# \#} p<0.01$ compared with AD

in response to cholesterol accumulation. Interestingly, the autophagic flux in HepG2 cells was dose-dependently blocked by PGE2, as suggested by the accumulation of both the LC3B and p62 proteins. In contrast, BBR treatment in PGE2-treated cells restored the autophagic flux (Fig. 6e). We also observed that PGE2 activated AKT phosphorylation and that BBR inhibited this process (Fig. 6e). These data suggested that PG metabolites might be the mediators that inhibited the autophagy process in hepatocytes, which was efficiently assuaged by BBR treatment.

\section{Discussion}

Hepatic cholesterol overloading blocks autophagic flux via the activation of $\mathrm{AKT} / \mathrm{mTOR}$ signaling. Here, we found that $\mathrm{BBR}$ treatment down-regulated the phosphorylation of AKT/mTOR and ameliorated the blockade of hepatic autophagic flux (Fig. 7). This effect was connected with the ability of BBR to reduce cellular cholesterol loading, to inhibit cholesterol trafficking toward the PM, and to mitigate COX2-mediated PG synthesis, which together led to the inhibition of $\mathrm{AKT} / \mathrm{mTOR}$ phosphorylation.

The cellular cholesterol level is important for autophagy regulation ${ }^{20,34}$. In hepatic cells, we found that FC accumulation impaired autolysosome function and clearance, as evidenced by increase of p62 proteins and the accumulation of merged yellow puncta in cells expressing the RFP-GFP-LC3 fusion protein. This finding is in agreement with previous observations by Liu et al. ${ }^{34}$ and Wang et al. ${ }^{20}$. However, the response to cholesterol appears to be cell type-specific. In macrophages, unlike in hepatocytes, cholesterol accumulation induced autophagy to attenuate cholesterol toxicity by increasing the cholesterol efflux ${ }^{35}$. One important finding of our study is that BBR is capable of reversing the blockade of autophagic flux caused by cholesterol overloading in hepatocytes with decreased p62 protein levels. This effect was consistently observed both in vitro and in vivo.

The AKT/mTOR axis is a master signaling pathway in autophagy regulation ${ }^{30}$. We found that cholesterol overloading promoted AKT phosphorylation at the PM, which is a common mechanism of AKT signaling ${ }^{36}$. BBR treatment attenuated the translocation of AKT toward the PM as well as its phosphorylation. Our data provided evidences that BBR regulated the AKT/mTOR phosphorylation through three ways. First, BBR reduces the overall exogenous hepatic cholesterol level through inhibiting intestinal cholesterol absorption ${ }^{17,18}$. BBR was previously shown to inhibit the esterification of FC by ACAT2 in intestinal epithelium cells and to interfere with luminal cholesterol micelle formation ${ }^{17}$. AD consumption caused six-fold increase of CE in mouse livers, whereas BBR treatment reduced the hepatic CE level to about two-fold higher compared with that in mice fed with a regular chow diet. As a result, the blockade of autophagic flux caused by HCD was greatly ameliorated by BBR treatment. Ezetimibe is an even more potent inhibitor of intestinal cholesterol absorption ${ }^{22}$, and it almost normalized the hepatic cholesterol content to a level similar to that in mice fed with chow diet. Additionally, ezetimibe treatment produced a similar amelioration of the cholesterol-induced autophagic flux blockade in mouse livers.

Second, BBR plays a direct role in regulating cholesterol trafficking in hepatocytes. Upon cholesterol overloading, the FC rapidly distributed to the PM, which is a reservoir for cholesterol and is where AKT phosphorylation activated $^{25}$. Notably, depletion of FC at the PM would decrease the phosphorylation of AKT and $\mathrm{mTOR}^{31,37}$. $\mathrm{BBR}$ treatment decreased both the expression of SCP2 in hepatocytes and the PM cholesterol level, as evidenced by the filipin staining. Autophagy activation by itraconazole was reported to occur through inhibiting SCP2 expression, reducing cholesterol in the $\mathrm{PM}$, and inhibiting phosphorylation of $\mathrm{AKT} / \mathrm{mTOR}^{34}$. Although BBR treatment decreased cholesterol transportation to the PM, cholesterol did not accumulate in organelles such as the $\mathrm{ER}$, endosomes, or lysosomes, partly due to a simultaneous activation of CYP7A1, which is responsible for the conversion of cholesterol into bile acids. The capability of BBR to induce CYP7A1 was also found in both a human hepatoma cell line ${ }^{38}$ and rodents ${ }^{39,40}$. Moreover, the induction of CYP7A1 expression by genetic modulation or cholestyramine treatment resulted in the activation of autophagic flux in hepatocytes ${ }^{20}$.

Third, BBR affects the phosphorylation of AKT through the regulation of PG metabolism. PGH2 is converted from 

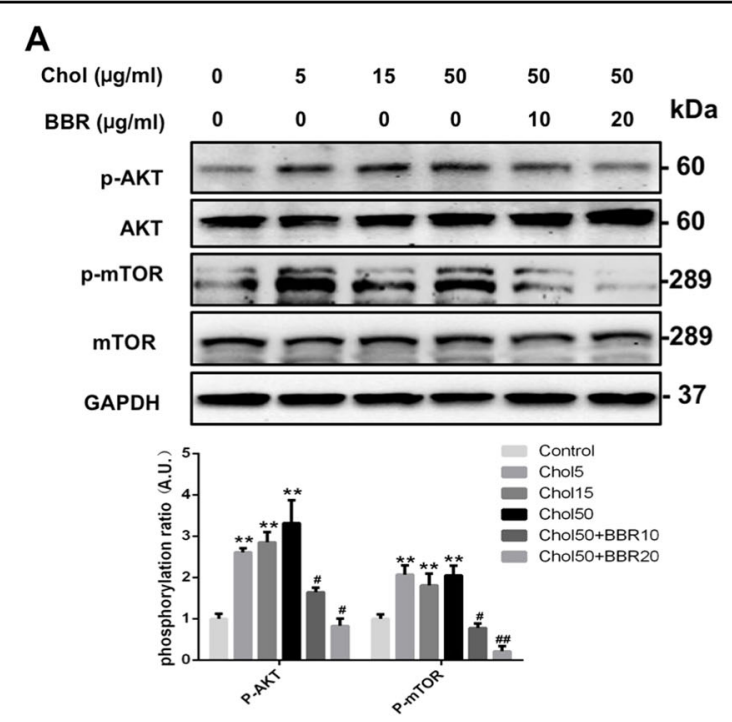

B

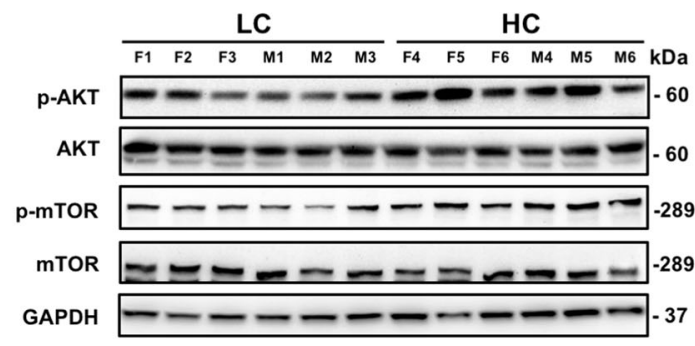

D

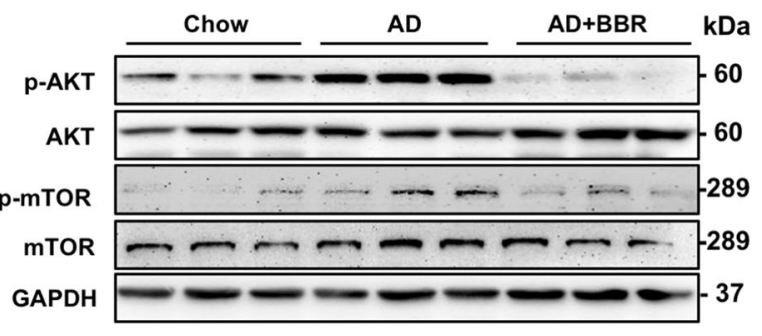

E
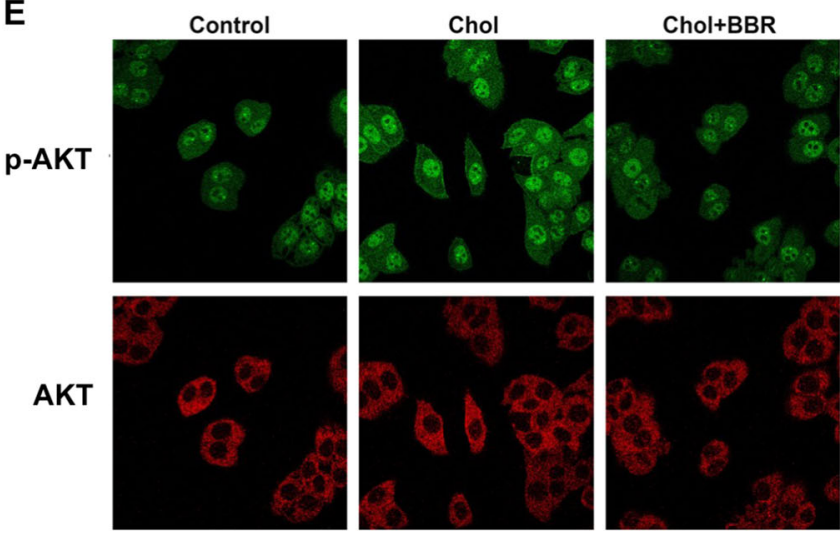

C
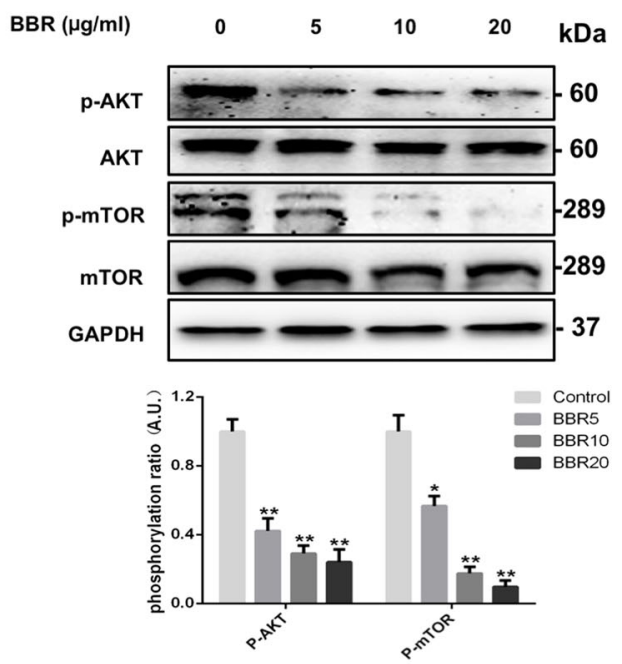
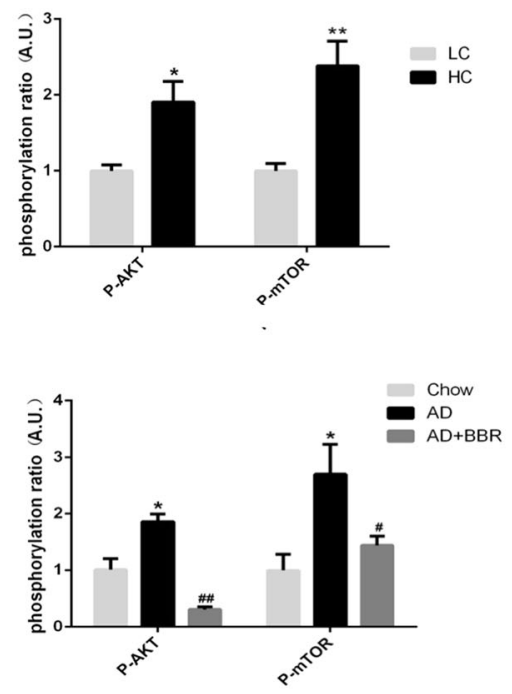

Fig. 4 (See legend on next page.) 
(see figure on previous page)

Fig. 4 Effect of BBR on AKT and mTOR phosphorylation. a Expression of phosphorylated AKT and mTOR in HepG2 cells treated with cholesterol (Chol) or cholesterol + berberine (BBR) for $24 \mathrm{~h}$. ${ }^{*} p<0.01$ compared with control. ${ }^{*} p<0.05$ and ${ }^{\# \#} p<0.01$ compared with cholesterol 50 . b Hepatic expression of phosphorylated AKT and mTOR protein levels between patients with high cholesterol level $(\mathrm{HC})$ and those with low cholesterol level $(\mathrm{LC})$ in their livers. ${ }^{*} p<0.05$ and ${ }^{* *} p<0.01$ for the HC group compared with the LC group. $\mathbf{c}$ Expression of phosphorylated AKT and mTOR in HepG2 cells treated with different concentrations of BBR for $24 \mathrm{~h} .{ }^{*} p<0.05$ and ${ }^{* *} p<0.01$ compared with control. $\mathbf{d}$ Expression of phosphorylated AKT and mTOR in the livers of mice fed with different diets. ${ }^{*} p<0.05$ compared with chow diet. ${ }^{\#} p<0.05$ and ${ }^{\# \#} p<0.01$ compared with AD. e Confocal microscopy images of p-AKT activation in HepG2 cells treated with cholesterol (Chol: $50 \mu \mathrm{g} / \mathrm{ml})$ alone or with cholesterol $(\mathrm{Chol}: 50 \mu \mathrm{g} / \mathrm{ml})+\mathrm{BBR}$ $(20 \mu \mathrm{g} / \mathrm{ml})$ for $8 \mathrm{~h}$; pervanadate $(50 \mu \mathrm{M})$, a strong AKT dephosphorization inhibitor, was used as a positive control

AA by cyclooxygenase and then further metabolized into D, E, F, and I series PGs by PG-synthases and isomerases. As the rate-limiting enzyme in PG synthesis, COX2 is induced by cytokines, growth factors, and tumors ${ }^{41}$. The AD consumption is known to induce hepatic inflammation via the upregulation of pro-inflammatory cytokines, such as tumor necrosis factor- $\alpha$ (TNF $\alpha)$, interleukin (IL) $1 \beta$, and IL6. As a result, COX2-mediated PG metabolites are elevated in the liver of mice fed with AD. We, for the first time, showed that BBR treatment inhibited hepatic COX2 expression and reduced PG metabolite accumulation. BBR treatment has been previously reported to decrease obesity-induced liver inflammation ${ }^{42}$ and to reduce ER stress during liver steatosis ${ }^{16}$. Increase of $\mathrm{PGE}^{43,44}$ as well as TNF $\alpha^{45}$ and IL1 $\beta^{46}$ was shown to induce COX2 expression facilitating the activation of AKT. Here, we observed that PGE2 dose-dependently activated AKT phosphorylation and blocked the autophagic flux in HepG2 cells, which was reversed by BBR treatment.

Collectively, the present study revealed that BBR could improve autophagic flux in hepatocytes by decreasing AKT/mTOR phosphorylation through the mechanisms depicted in Fig. 7. The results of our study provided a novel mechanism for how BBR protects liver through the activation of autophagy process, in addition to its role in regulating the cholesterol level. BBR might be a therapeutic option for treating conditions in which the liver is burdened by cholesterol loading.

\section{Materials and Methods}

\section{Regents and materials}

Berberine chloride (BBR), $\mathrm{CQ}$, wheat germ agglutinin (WGA), water-soluble cholesterol (in methyl-beta-cyclodextrin), prostaglandin E (PGE2), and dimethyl sulfoxide (DMSO) were purchased from Sigma-Aldrich (Supplementary Table 2). All information concerning the antibodies used in this study are listed Supplementary Table 3.

\section{Animal studies}

Mouse food for AD (containing 1.25\% cholesterol and $0.5 \%$ cholic acid) and HCD (containing $1.25 \%$ cholesterol) were purchased from Trophic Animal Feed High-Tech Co. Ltd (Nantong, China). Adult male C57BL/6 mice (8week-old, Shanghai SLAC Laboratory Animal Co., Ltd, Shanghai, China) were randomly allocated to groups fed with different diets ( $n=5$ mice/group): chow diet, $\mathrm{AD}$, $\mathrm{HCD}, \mathrm{AD}+\mathrm{BBR}(100 \mathrm{mg} / \mathrm{kg} /$ day, by gavage), AD + ezetimibe $(5 \mathrm{mg} / \mathrm{kg} /$ day, by gavage), or HCD + BBR (100 mg/ $\mathrm{kg} /$ day, by gavage) for 8 weeks. Upon sacrifice, blood samples and liver tissues were collected. All the experiment protocols were approved by the Animal Use and Care Committee of Shanghai East Hospital, Tongji University School of Medicine.

\section{Cell culture}

HepG2 cells were cultured in Dulbecco's modified Eagle's medium containing $10 \%$ fetal bovine serum. Working solution of pervanadate was prepared using a previously described method ${ }^{47}$. Before any treatment, equal numbers of cells were plated in six-well plates and cultured until all wells reached $\sim 70 \%$ confluence. These cells were then washed with cold phosphate-buffered saline (PBS) at least twice and subjected to BBR, CQ, cholesterol, PGE2, or pervanadate treatment. Finally, the cells were either harvested for western blot analysis or fixed for immunofluorescence staining.

\section{mRFP-GFP-LC3 adenovirus transduction}

The mRFP-GFP-LC3 adenovirus was obtained from HanBio Inc. (Shanghai, China). HepG2 cells were infected with adenoviral particles at a multiplicity of infection (MOI) of 10 for $24 \mathrm{~h}$. After complete adenovirus transduction, the cells were washed with PBS and subjected to $\mathrm{CQ}$, cholesterol, or BBR treatment. Following the treatment, $4 \%$ paraformaldehyde was used to fix the cells, and a Zeiss LSM-710 confocal microscope was used to observe the number of GFP-positive and mRFP-positive dots.

\section{Lentivirus transduction}

A lentivirus encoding prostaglandin-endoperoxide synthase 2 (PTGS2), also known as COX2, was purchased from GeneChem (Shanghai, China). Before transduction, equal numbers of HepG2 cells were plated in six-well 
A

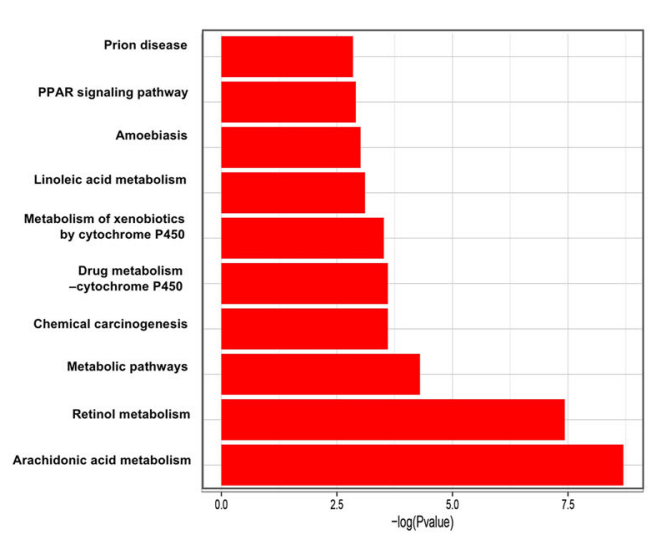

C

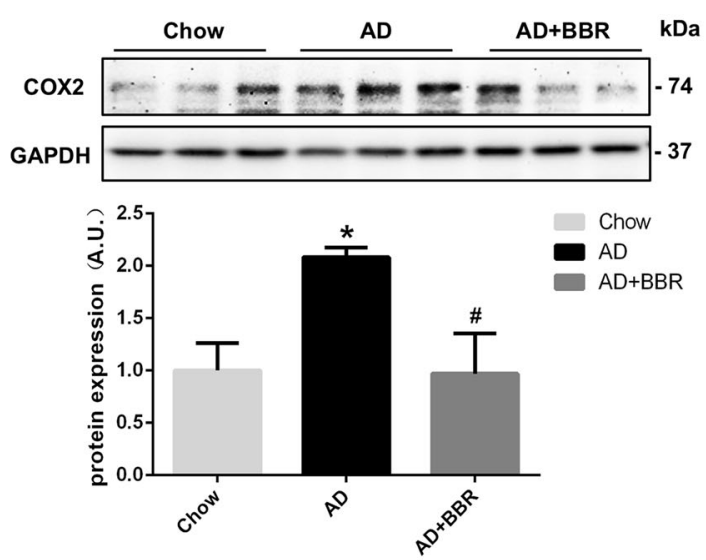

E

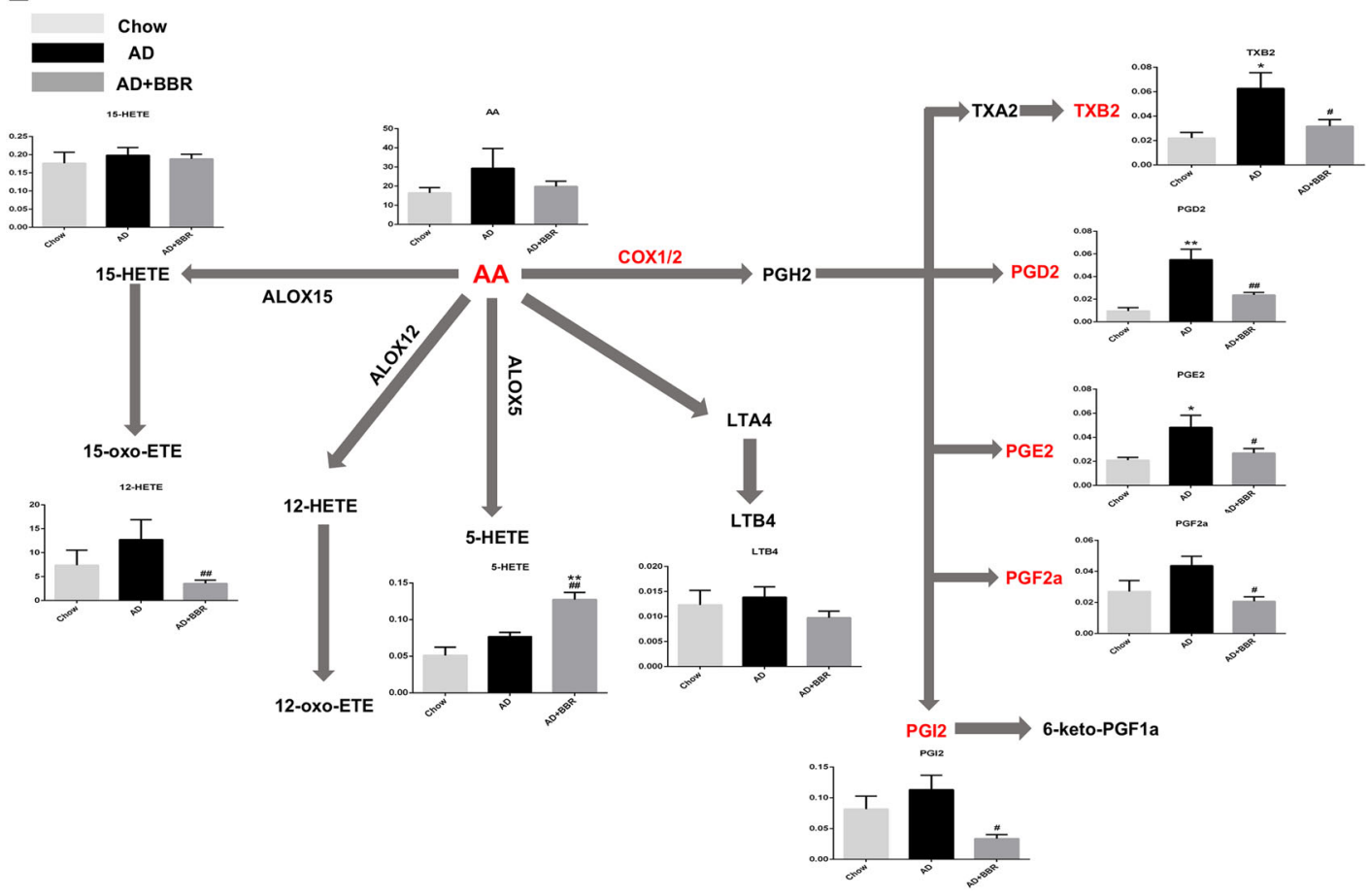

B

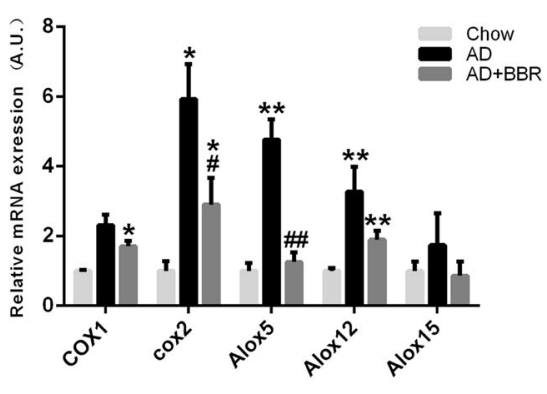

D

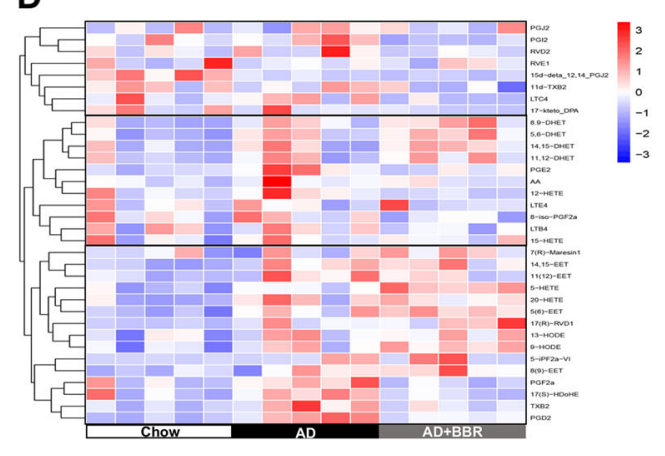

Fig. 5 (See legend on next page.) 
(see figure on previous page)

Fig. 5 Regulation of hepatic COX2/prostaglandin metabolism by BBR. a KEGG analysis of the top 10 pathways in the livers of mice fed with atherogenic diet (AD) alone or with berberine (AD + BBR). $\mathbf{b}$ Expression of key genes involved in the hepatic metabolism of arachidonic acid and prostaglandin metabolites. c Changes of the COX2 protein levels in the livers of mice fed with different diets. ${ }^{*} p<0.05$ compared with chow diet. ${ }^{*} p$ $<0.05$ compared with AD. d Heatmap show of arachidonic acid and prostaglandin metabolites in the livers of mice fed with chow, AD, and AD + BBR. e Levels of arachidonic acid and prostaglandin metabolites among groups (unit: ng/mg liver). ${ }^{*} p<0.05$ and ${ }^{* *} p<0.01$ compared with chow diet. ${ }^{\#} p<0.05$ and ${ }^{\# \#} p<0.01$ compared with AD

plates. When the cells reached $\sim 50 \%$ confluence, they were infected with lentivirus particles at an MOI of 10 for $16 \mathrm{~h}$. To obtain stable clones, the infected cell lines were selected by puromycin $(5 \mu \mathrm{g} / \mathrm{ml})$ for 2 weeks. Once established, these stable clonal cells were subjected to either BBR treatment or mRFP-GFP-LC3 adenovirus transduction for further experiments.

\section{H\&E staining and electron microscopy examination}

Paraffin-embedded livers were cut into sections of $5 \mu \mathrm{m}$ in thickness and stained with H\&E as previously described $^{48}$. A JEOL-1010 transmission electron microscope was used for examining the liver tissue samples as previously described ${ }^{48}$.

\section{Immunofluorescence staining}

HepG2 cells were fixed with $4 \%$ paraformaldehyde and then blocked with a solution containing $5 \%$ bovine serum albumin and $0.02 \%$ Triton-X 100. After incubation overnight at $4{ }^{\circ} \mathrm{C}$ with primary antibodies against LC3B, p62, COX2, AKT, p-AKT, or LAMP-1, cells were washed with PBS and then further incubated for 90 min away from light with an appropriate fluorescence-conjugated secondary antibody. Nuclei was stained with DAPI (Invitrogen, Carlsbad, CA, USA). Finally, images of the cells were acquired by a fluorescence microscope (Axio Imager M2; Carl Zeiss Microscopy GmbH, Germany) or a confocal microscope (LSM-710; Carl Zeiss Microscopy $\mathrm{GmbH})$.

\section{Filipin staining}

Filipin III $(2 \mathrm{mg})$ was dissolved in $800 \mu \mathrm{l}$ of DMSO as a $50 \times$ stock solution. After treatment, HepG2 Cells were washed twice with cold PBS and fixed with $4 \%$ paraformaldehyde for $15 \mathrm{~min}$. The samples were then stained away from light for $2 \mathrm{~h}$ with a $50 \mathrm{\mu g} / \mathrm{ml}$ filipin III working solution in PBS. Images were acquired using a Zeiss LSM710 confocal microscope.

\section{Quantification of arachidonic acid metabolites by LC-MS}

Liver tissue samples $(50 \mathrm{mg}$ each) from mice in the chow, $\mathrm{AD}$, and $\mathrm{AD}+\mathrm{BBR}$ diet groups were subjected to a determination of AA metabolites by LC-MS according to previously described procedures ${ }^{49}$.

\section{Western blot analysis}

Mouse or human liver homogenates and HepG2 cell lysates were prepared, and their protein concentrations were determined with the BCA method. Protein samples $(20 \mu \mathrm{g}$ each) were loaded and separated on 10\% SDSPAGE. After the proteins were transferred onto polyvinylidene fluoride membranes (Millipore, Billerica, MA, USA), the membranes were blocked, incubated overnight at $4{ }^{\circ} \mathrm{C}$ with an appropriate primary antibody, and then incubated for $2 \mathrm{~h}$ at room temperature with an appropriate secondary antibody. Protein brands were visualized using ECL Plus reagent (Beyotime, Shanghai, China). Each experiment was replicated at least three times, and the acquired images were quantified by ImageJ software.

\section{RNA-seq}

Total RNA was extracted from liver tissues using TRIzol reagent (Invitrogen). The corresponding cDNA libraries were constructed using the TruSeq Stranded Total RNA LT Sample Prep Kit (Illumina, Santiago, CA, USA) by Genergy Biological Technology Limited Co. (Shanghai, China) and sequenced with Illumina Hiseq3000. RNA-Seq reads were aligned to the reference data downloaded from UCSC (version hg19), and the RPKM method was utilized to normalize the reads that exclusively mapped to a gene, to quantify the transcript levels ${ }^{50,51}$.

\section{Quantitative real-time PCR}

Total RNA from mouse livers were extracted by TRIzol reagent (Invitrogen), and then reverse-transcribed into cDNA using a High Capacity cDNA Reverse Transcription Kit (Applied Biosystems Foster City, CA, USA). Power Mix Sybr Green Master Mix (Applied Biosystems) was used for quantitative real-time $\mathrm{PCR}^{52}$ to determine the gene expressions of key enzymes involved in the AA metabolism (primer sequences are available upon request). The relative mRNA expression was then calculated with the $\triangle \Delta \mathrm{Ct}$ method using GAPDH as the internal control.

\section{Statistics}

All the data are presented as the mean \pm SEM. The comparisons among multiple groups were performed using a one-way ANOVA with a post-hoc test (Tukey's 


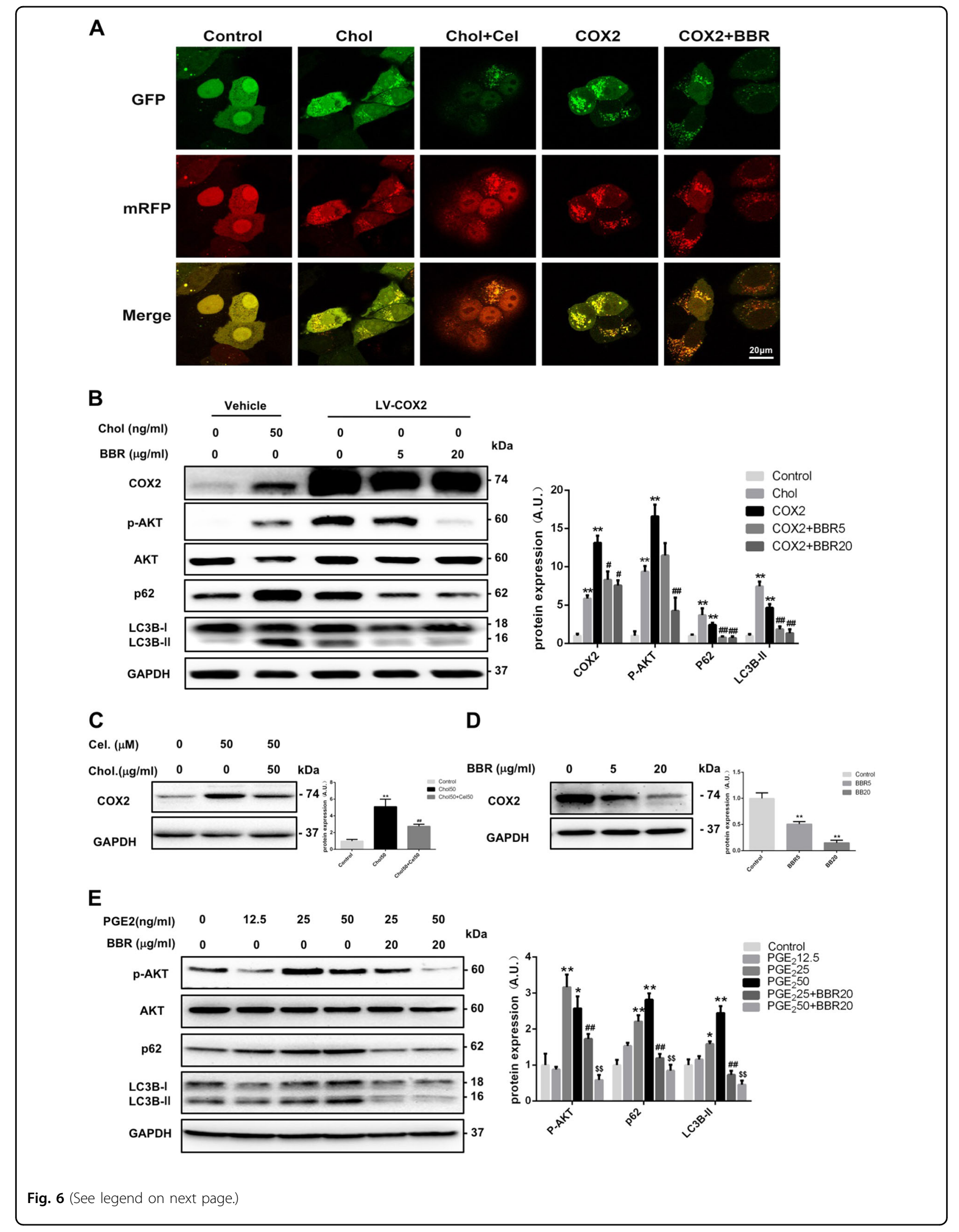


(see figure on previous page)

Fig. 6 COX2 expression and autophagic flux in BBR-treated HepG2 cells. a Confocal microscopy examination of HepG2 cells expressing a tandem GFP-RFP-LC3 fusion protein that were treated with cholesterol (Chol, $50 \mu \mathrm{g} / \mathrm{ml}$ ) with/without celecoxib (Cel; $50 \mu \mathrm{M})$, or subjected to lentivirus-mediated overexpression of COX2 protein with/without berberine (BBR, $20 \mu \mathrm{g} / \mathrm{ml})$ treatment for $24 \mathrm{~h}$. $\mathbf{b}$ Protein levels of COX2, p-AKT, LC3II, and p62 in HepG2 cells treated with cholesterol or lentivirus-mediated overexpression of COX2 with BBR for $24 \mathrm{~h} .{ }^{* *} p<0.01$ compared with control. ${ }^{\#} p<0.05$ and ${ }^{\# \#} p<0.01$ compared with LV-COX2. c, d Expression of COX2 protein in HepG2 cells treated with cholesterol, cholesterol + Cel, or BBR for $24 \mathrm{~h} .{ }^{* *} p<0.01$ compared with control. ${ }^{\# \#} p<0.01$ compared with cholesterol. e Expression of p-AKT, p62, and LC3Il proteins in HepG2 cells treated with different concentrations of PGE2 alone or together with BBR for $24 \mathrm{~h} .{ }^{*} p<0.05$ and ${ }^{* *} p<0.01$ compared with control. ${ }^{\# \#} p<0.01$ compared with $\mathrm{PGE}_{2}$ 25. ${ }^{\$} p<0.01$ compared with $\mathrm{PGE}_{2} 50$

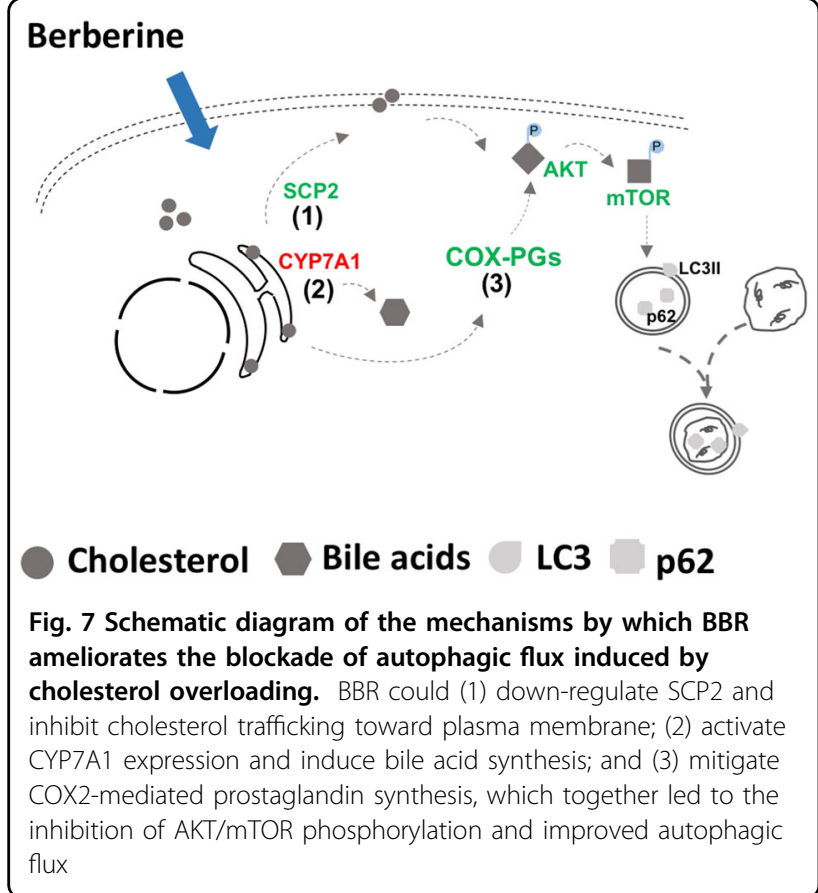

multiple comparison test). A $p$-value of less than 0.05 was considered statistically significant. All the statistical tests were performed with IBM SPSS statistics 21.0 software.

\section{Acknowledgements}

This work was supported by the National Science Fund for Outstanding Young Scholars (81722040); the National Natural Science Foundation of China (81770626, 81573174, 81570574); the Key Specialty Construction Project of Pudong Health and Family Planning Commission of Shanghai (PWZzk2017-10); the Outstanding Youth Fund of Jiangsu Province (SBK2014010296); the Research Project of Chinese Ministry of Education (213015A); the Priority Academic Program Development of Jiangsu Higher Education Institutions (PAPD), the Flagship Major Development of Jiangsu Higher Education Institutions; and the Open Project Program of the State Key Laboratory of Environmental Chemistry and Ecotoxicology (KF2015-01).

\section{Authors' contributions}

A.G. and Z.J.: designed the study, interpreted the data, and wrote the manuscript; S.H., Q.L., H.H., Y.J., W.S. and Q.W.: performed the experiments; S.H. and Q.L.: collected the data, performed the analyses, and wrote the manuscript. All authors approved the final version of the manuscript.

\section{Author details}

${ }^{1}$ Center of Gallbladder Disease, Shanghai East Hospital, Institute of Gallstone Disease, Tongji University School of Medicine, Shanghai, China. ${ }^{2}$ State Key
Laboratory of Reproductive Medicine, Institute of Toxicology, Nanjing Medical University, Nanjing, China. ${ }^{3}$ Key Laboratory of Modern Toxicology of Ministry of Education, School of Public Health, Nanjing Medical University, Nanjing, China. ${ }^{4}$ Yucai High School, Shanghai, China

\section{Conflict of interest}

The authors declare that they have no conflict of interest.

\section{Publisher's note}

Springer Nature remains neutral with regard to jurisdictional claims in published maps and institutional affiliations.

Supplementary Information accompanies this paper at (https://doi.org/ 10.1038/s41419-018-0890-5).

Received: 27 April 2018 Revised: 12 July 2018 Accepted: 17 July 2018 Published online: 01 August 2018

\section{References}

1. Brown, D. F. Blood lipids and lipoproteins in atherogenesis. Am. J. Med. 46, 691-704 (1969).

2. Kannel, W. B. Lipid profile and the potential coronary victim. Am. J. Clin. Nutr. 24, 1074-1081 (1971).

3. Go, A. S. et al. Heart disease and stroke statistics-2013 update: a report from the American Heart Association. Circulation 127, e6-e245 (2013).

4. National Cholesterol Education Program Expert Panel on Detection, Evaluation \& Treatment of High Blood Cholesterol in Adults. Third Report of the National Cholesterol Education Program (NCEP) Expert Panel on Detection, Evaluation, and Treatment of High Blood Cholesterol in Adults (Adult Treatment Panel III) final report. Circulation 106, 3143-3421 (2002).

5. Sozen, E. \& Ozer, N. K. Impact of high cholesterol and endoplasmic reticulum stress on metabolic diseases: an updated mini-review. Redox Biol. 12, 456-461 (2017).

6. Hendrikx, T., Walenbergh, S. M., Hofker, M. H. \& Shiri-Sverdlov, R. Lysosomal cholesterol accumulation: driver on the road to inflammation during atherosclerosis and non-alcoholic steatohepatitis. Obes. Rev. 15, 424-433 (2014).

7. Gan, L. T. et al. Hepatocyte free cholesterol lipotoxicity results from JNK1mediated mitochondrial injury and is HMGB1 and TLR4-dependent. J. Hepatol. 61, 1376-1384 (2014).

8. Ueno, T. \& Komatsu, M. Autophagy in the liver: functions in health and disease Nat. Rev. Gastroenterol. Hepatol. 14, 170-184 (2017).

9. Madrigal-Matute, J. \& Cuervo, A. M. Regulation of liver metabolism by autophagy. Gastroenterology 150, 328-339 (2016).

10. Mizushima, N., Levine, B., Cuervo, A. M. \& Klionsky, D. J. Autophagy fights disease through cellular self-digestion. Nature 451, 1069-1075 (2008).

11. Singh, R. et al. Autophagy regulates lipid metabolism. Nature 458, 1131-1135 (2009).

12. Liu, K. \& Czaja, M. J. Regulation of lipid stores and metabolism by lipophagy. Cell Death Differ. 20, 3-11 (2013).

13. Abidi, P., Zhou, Y., Jiang, J. D. \& Liu, J. Extracellular signal-regulated kinasedependent stabilization of hepatic low-density lipoprotein receptor mRNA by herbal medicine berberine. Arterioscler. Thromb. Vasc. Biol. 25, 2170-2176 (2005).

14. Kong, W. et al. Berberine is a novel cholesterol-lowering drug working through a unique mechanism distinct from statins. Nat. Med. 10, 1344-1351 (2004). 
15. Hao, X. et al. Berberine ameliorates pro-inflammatory cytokine-induced endoplasmic reticulum stress in human intestinal epithelial cells in vitro. Inflammation 35, 841-849 (2012).

16. Zhang, Z. et al. Berberine prevents progression from hepatic steatosis to steatohepatitis and fibrosis by reducing endoplasmic reticulum stress. Sci. Rep. 6, 20848 (2016)

17. Wang, $Y$. et al. Berberine decreases cholesterol levels in rats through multiple mechanisms, including inhibition of cholesterol absorption. Metabolism 63 1167-1177 (2014).

18. Wang, Y. et al. Berberine and plant stanols synergistically inhibit cholesterol absorption in hamsters. Atherosclerosis 209, 111-117 (2010).

19. Deng, $Y$. et al. Berberine attenuates autophagy in adipocytes by targeting BECN1. Autophagy 10, 1776-1786 (2014).

20. Wang, Y. et al. Targeting the enterohepatic bile acid signaling induces hepatic autophagy via a CYP7A1-AKT-mTOR axis in mice. Cell. Mol. Gastroenterol. Hepatol. 3, 245-260 (2017).

21. Manning, B. D. \& Toker, A. AKT/PKB signaling: navigating the network. Cell 169, 381-405 (2017).

22. Altmann, S. W. et al. Niemann-Pick C1 Like 1 protein is critical for intestinal cholesterol absorption. Science 303, 1201-1204 (2004).

23. Klionsky, D. J. et al. Guidelines for the use and interpretation of assays for monitoring autophagy (3rd edition). Autophagy 12, 1-222 (2016).

24. Ikonen, E. Cellular cholesterol trafficking and compartmentalization. Nat. Rev. Mol. Cell Biol. 9, 125-138 (2008).

25. Maxfield, F. R. \& Wustner, D. Intracellular cholesterol transport. J. Clin. Invest. 110, 891-898 (2002)

26. laea, D. B. \& Maxfield, F. R. Cholesterol trafficking and distribution. Essays Biochem. 57, 43-55 (2015).

27. Puglielli, L., Rigotti, A., Greco, A. V., Santos, M. J. \& Nervi, F. Sterol carrier protein2 is involved in cholesterol transfer from the endoplasmic reticulum to the plasma membrane in human fibroblasts. J. Biol. Chem. 270, 18723-18726 (1995).

28. Holtta-Vuori, M. et al. MLN64 is involved in actin-mediated dynamics of late endocytic organelles. Mol. Biol. Cell 16, 3873-3886 (2005).

29. Carstea, E. D. et al. Niemann-Pick C1 disease gene: homology to mediators of cholesterol homeostasis. Science 277, 228-231 (1997).

30. Jung, C. H., Ro, S. H., Cao, J., Otto, N. M. \& Kim, D. H. mTOR regulation of autophagy. FEBS Lett. 584, 1287-1295 (2010).

31. Lasserre, R. et al. Raft nanodomains contribute to Akt/PKB plasma membrane recruitment and activation. Nat. Chem. Biol. 4, 538-547 (2008).

32. Zhuang, L., Kim, J., Adam, R. M., Solomon, K. R. \& Freeman, M. R. Cholesterol targeting alters lipid raft composition and cell survival in prostate cancer cells and xenografts. J. Clin. Invest. 115, 959-968 (2005).

33. Andjelkovic, M. et al. Activation and phosphorylation of a pleckstrin homology domain containing protein kinase (RAC-PK/PKB) promoted by serum and protein phosphatase inhibitors. Proc. Natl. Acad. Sci. USA 93, 5699-5704 (1996).

34. Liu, R. et al. Itraconazole suppresses the growth of glioblastoma through induction of autophagy: involvement of abnormal cholesterol trafficking. Autophagy 10, 1241-1255 (2014).
35. Liao, X. et al. Macrophage autophagy plays a protective role in advanced atherosclerosis. Cell Metab. 15, 545-553 (2012).

36. Andjelkovic, M. et al. Role of translocation in the activation and function of protein kinase B. J. Biol. Chem. 272, 31515-31524 (1997).

37. Mollinedo, F. \& Gajate, C. Lipid rafts as major platforms for signaling regulation in cancer. Adv. Biol. Regul. 57, 130-146 (2015).

38. Sun, R. et al. Orally administered berberine modulates hepatic lipid metabolism by altering microbial bile acid metabolism and the intestinal FXR signaling pathway. Mol. Pharmacol. 91, 110-122 (2017).

39. Guo, Y., Zhang, Y., Huang, W., Selwyn, F. P. \& Klaassen, C. D. Dose-response effect of berberine on bile acid profile and gut microbiota in mice. BMC Complement. Altern. Med. 16, 394 (2016).

40. Gu, S. et al. A metabolomic and pharmacokinetic study on the mechanism underlying the lipid-lowering effect of orally administered berberine. Mol. Biosyst. 11, 463-474 (2015)

41. Williams, C. S. \& DuBois, R. N. Prostaglandin endoperoxide synthase: why two isoforms? Am. J. Physiol. 270, G393-G400 (1996).

42. Guo, T. et al. Berberine ameliorates hepatic steatosis and suppresses liver and adipose tissue inflammation in mice with diet-induced obesity. Sci. Rep. $\mathbf{6}$, 22612 (2016).

43. Vo, B. T. et al. TGF-beta effects on prostate cancer cell migration and invasion are mediated by PGE2 through activation of PI3K/AKT/mTOR pathway. Endocrinology 154, 1768-1779 (2013).

44. Jana, S., Chatterjee, K., Ray, A. K., DasMahapatra, P. \& Swarnakar, S. Regulation of matrix metalloproteinase-2 activity by COX-2-PGE2-pAKT axis promotes angiogenesis in endometriosis. PLOS ONE 11, e0163540 (2016).

45. Hai, L., Kawarabayashi, Y., Imai, Y., Honda, A. \& Inoue, R. Counteracting effect of TRPC1-associated $\mathrm{Ca}+$ influx on TNF-alpha-induced COX-2-dependent prostaglandin E2 production in human colonic myofibroblasts. Am. J. Physiol. Gastrointest. Liver Physiol. 301, G356-G367 (2011).

46. Lou, Y. et al. Paeonol inhibits IL-1beta-induced inflammation via PI3K/Akt/NFkappaB pathways: in vivo and vitro studies. Inflammation 40, 1698-1706 (2017).

47. Huyer, G. et al. Mechanism of inhibition of protein-tyrosine phosphatases by vanadate and pervanadate. J. Biol. Chem. 272, 843-851 (1997).

48. Liu, Q. et al. Organochloride pesticides impaired mitochondrial function in hepatocytes and aggravated disorders of fatty acid metabolism. Sci. Rep. 7, 46339 (2017).

49. Huang, Y. et al. Mass spectrometry-based metabolomic profiling identifies alterations in salivary redox status and fatty acid metabolism in response to inflammation and oxidative stress in periodontal disease. Free Radic. Biol. Med. 70, 223-232 (2014).

50. Mortazavi, A., Williams, B. A., McCue, K., Schaeffer, L. \& Wold, B. Mapping and quantifying mammalian transcriptomes by RNA-Seq. Nat. Methods 5, 621-628 (2008).

51. Liu, Y. et al. SUMO-specific protease 3 is a key regulator for hepatic lipid metabolism in non-alcoholic fatty liver disease. Sci. Rep. 6, 37351 (2016).

52. Liu, Q. et al. Organochloride pesticides modulated gut microbiota and influenced bile acid metabolism in mice. Environ. Pollut. 226, 268-276 (2017). 